\title{
RELAÇÃO ENTRE RESILIÊNCIA E BURNOUT: PROMOÇÃO DA SAÚDE MENTAL E OCUPACIONAL DOS ENFERMEIROS
}

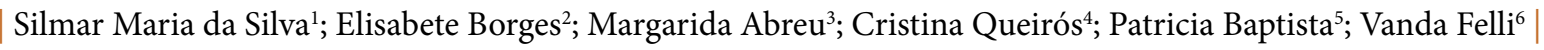

\section{RESUMO}

INTRODUÇÃO: Os enfermeiros exercem a sua atividade num ambiente de trabalho fértil em fatores que favorecem o aumento dos níveis de stresse no trabalho. Estes afetam o trabalhador e as organizações, podendo a resiliência ser um fator protetor.

OBJETIVO: Conhecer os níveis de Resiliência e Burnout de enfermeiros, sua variação em função de caracteristicas sociodemográficas e profissionais e a relação entre ambos, no sentido de verificar se a Resiliência pode ajudar na promoção da saúde mental e ocupacional dos enfermeiros. METODOLOGIA: Questionário de Caracterização Sociodemográfica e Profissional, Escala de Resiliência e Maslach Burnout Inventory (HSS). Participaram de forma anónima e voluntária 200 enfermeiros de hospitais públicos da área metropolitana do Porto, sendo $70 \%$ do sexo feminino, com idades entre 25 e 57 anos ( $M=33,8$ anos), $43 \%$ casados ou em união de facto, $36 \%$ com filhos, $82 \%$ com licenciatura, $76 \%$ com vinculo definitivo, $80 \%$ trabalha em turno rotativo, média 10,6 anos de experiência na profissão e média de 6,6 anos de tempo na instituição.

RESULTADOS: Encontraram-se niveis de moderada exaustão emocional, baixos valores de despersonalização e elevados valores de realização pessoal e de Resiliência. Existem correlações negativas entre exaustão emocional e resiliência, e correlações positivas entre realização pessoal e resiliência. A análise comparativa evidenciou o turno com diferenças significativas na despersonalização, tendo valores mais elevados nos trabalhadores de turno rotativo. A resiliencia explica negativamente $8 \%$ da exaustão emocional e positivamente $26 \%$ da realização pessoal, surgindo apenas $5 \%$ da amostra com Burnout elevado e $12 \%$ com resiliência reduzida.

CONCLUSÃO: A resiliência pode ajudar a reduzir a vulnerabilidade dos enfermeiros ao Burnout, pois elevada resiliência está relacionada com menos stresse, protegendo do Burnout.Os resultados alertam para a prevenção do Burnout, nomeadamente no que se refere aos turnos, pois estes comprometem o comportamento resiliente e aumentam a vulnerabilidade ao Burnout.

\section{PALAVRAS-CHAVE: Saúde do trabalhador; Enfermagem; Burnout; Resiliência psicológica}

\section{RESUMEN}

"Relación entre resiliencia y burnout: Promoción de la salud mental y ocupacional de los enfermeros"

INTRODUCCIÓN: Los enfermeros trabajan en contextos estresantes, lo que los afecta a eles y a las organizaciones. La Resiliencia puede ser un factor protector. OBJETIVO: Conocer los niveles de resiliencia y de agotamiento profesional (Burnout) de los enfermeros, su variación en función de las características sociodemográficas e profesionales y la relación entre ellos, para verificar si la resiliencia ayuda en la promoción de la salud mental y laboral de los enfermeros. METODOLOGÍA: Cuestionario de caracterización demográfica y profesional, Escala de Resiliencia y Maslach Burnout Inventory (HSS). Participaron de forma anónima y voluntaria, 200 enfermeros de hospitales públicos de Porto: $70 \% \mathrm{mu}-$ jeres, con edad entre 25-57 años ( $M=33,8$ años), $43 \%$ casadas/con pareja, $36 \%$ con niños, $82 \%$ con grado, $76 \%$ con contrato laboral definitivo, $80 \%$ con cambio de horas laborales. Tenían 10,6 años de experiencia en la profesión y 6,6 años en la institución.

RESULTADOS: Fueran encontrados niveles moderados de agotamiento emocional, bajos valores de despersonalización y altos valores de realización personal y resiliencia. Hay correlaciones negativas entre agotamiento emocional y resiliencia, correlaciones positivas entre realización personal y resiliencia. El análisis comparativo demostró que los cambios laborales de hora afectan la despersonalización, con valores superiores en los trabajadores que cambian las horas. La resiliencia explica negativamente $8 \%$ de agotamiento emocional y positivamente $26 \%$ de realización personal. Solamente $5 \%$ de los participantes presentan Burnout elevado y $12 \%$ tienen resiliencia reducida.

CONCLUSIÓN: La resiliencia puede ayudar a reducir la vulnerabilidad de los enfermeros a lo agotamiento profesional, porque una elevada resiliencia se relaciona con menos tensión, protegiendo de lo agotamiento profesional. Los resultados advierten para la prevención de agotamiento profesional, sobretodo relacionado con los horarios laborales.

\section{DESCRIPTORES: Salud laboral; Enfermería; Agotamiento profesional; Resiliencia psicológica}

\section{ABSTRACT \\ "Relationship between resilience and burnout: Mental and oc- cupational health promotion among nurses"}

INTRODUCTION: Nurses work in stressful contexts, which affect them and the organizations. However, resilience can be a protective factor.

AIM: To know Resilience and Burnout levels among nurses, their variation according sociodemographic and professional characteristics, and the relationship between these variables, in order to verify if resilience can help to promote nurses' mental and occupational health.

METHODS: Questionnaire about sociodemographic and professional data, Resilience Scale and Maslach Burnout Inventory (HSS). Participated anonymously and voluntary 200 nurses of public hospitals from the metropolitan area of Oporto, being $70 \%$ female, $82 \%$ graduated, $76 \%$ with a definitive contract, $80 \%$ working in rotating shift. They have an average of 10.6 years of professional experience, and 6.6 years of time at the current institution.

RESULTS: We found moderate levels of emotional exhaustion, low values of depersonalization and high values of personal achievement and resilience. There are negative correlations between emotional exhaustion and resilience, and positive correlations between personal achievement and resilience. Comparative analysis showed that only work shifts present significant differences in depersonalization, with higher values among nurses with rotated work shifts. Resilience explains negatively $8 \%$ of emotional exhaustion and positively $26 \%$ of personal achievement. Only $5 \%$ of the participants present high Burnout levels and only $12 \%$ present reduced resilience levels.

CONCLUSION: Resilience can help to reduce the nurses' vulnerability to Burnout, since high resilience is related with less stress, protecting from Burnout. Results alerts for Burnout prevention, particularly related with work shifts.

\section{KEYWORDS: Occupational health; Nursing; Burnout; Resil- ience}

Submetido em 15-03-2016

Aceite em 20-11-2016

1 Enfermeira; Doutoranda em Ciências pela Universidade de São Paulo, Escola de Enfermagem, São Paulo, Brasil, silmarmaria@uol.com.br

2 Doutora em Enfermagem; Professora Adjunta na Escola Superior de Enfermagem do Porto, Porto, Portugal, elisabete@esenf.pt

3 Doutora em Enfermagem; Professora Coordenadora na Escola Superior de Enfermagem do Porto, Porto, Portugal, mabreu@esenf.pt

4 Doutora em Psicologia; Professora Auxiliar na Universidade do Porto, Faculdade de Psicologia e de Ciências da Educação, Porto, Portugal; cqueiros@fpce.up.pt

5 Doutora em Enfermagem; Professora Associada na Universidade de São Paulo, Escola de Enfermagem; Líder do Grupo de Pesquisa "Estudos sobre a saúde do trabalhador de enfermagem" na Universidade de São Paulo, Escola de Enfermagem, São Paulo, Brasil, pavanpati@usp.br

6 Doutora em Enfermagem; Professora Associada na Universidade de São Paulo, Escola de Enfermagem; Líder do Grupo de Pesquisa "Estudos sobre a saúde do trabalhador de enfermagem" na Universidade de São Paulo, Escola de Enfermagem, São Paulo, Brasil, vandaeli@usp.br

Citação: Silva, S. M., Borges, E., Abreu, M., Queirós, C., Baptista, P., \& Felli, V. (2016). Relação entre resiliência e burnout: Promoção da saúde mental e ocupacional dos enfermeiros. Revista Portuguesa de Enfermagem de Saúde Mental (16), 41-48. 


\section{INTRODUÇÃO}

Os enfermeiros exercem a sua atividade num ambiente de trabalho fértil em fatores que favorecem o aumento dos níveis de stresse no trabalho, tais como: prolongamento de turnos de trabalho, redução de recursos humanos, cumprimento de objetivos institucionais, relações de poder e hierárquicas extremamente demarcadas, exposição a agentes biológicos e químicos, entre outros (Maroco et al., 2016; Metzger, 2011; Ramos, Souza, Gonçalves, Pires \& Santos, 2014).

O stresse no trabalho refere-se a um processo de adaptação temporária ocupacional associada com sintomas fisiológicos, físicos e/ou cognitivos (Davey et al., 2016). No entanto, o Burnout é a resposta ao processo de stresse ocupacional crónico, ou seja, é o resultado de uma exposição prolongada ao stresse no trabalho causando um aumento na exaustão emocional, cinismo e ineficácia que representam as três dimensões de Burnout (Maslach \& Leiter, 2008). Neste sentido, a exaustão é compreendida como uma dimensão afetiva caracterizado por sentimentos de cansaço e esgotamento de energia emocional; o cinismo como uma dimensão interpessoal descrevendo as atitudes negativas em relação ao distanciamento e diferentes aspectos do próprio trabalho; e a ineficácia como uma dimensão que descreve o declínio nos sentimentos de competência, eficácia e produtividade no trabalho (Maslach \& Leiter, 2008). A incidência de Burnout ocorre, principalmente, quando não se reconhece o lado humano do trabalho, e quando existem grandes discrepâncias entre a natureza do trabalho e a natureza das pessoas (Maslach \& Leiter, 2008). Tanto o stresse no trabalho, quanto o Burnout são varáveis de contínua preocupação em enfermagem, afetando tanto os indivíduos e organizações (Davey et al., 2016). O Burnout não só tem apresentado impactos negativos na saúde mental e o bem-estar dos enfermeiros, mas também no desempenho e na qualidade da assistência ao paciente, no funcionamento e no comprometimento com a organização (Coates \& Howe, 2015; Khamisa, Oldenburg, Peltzer \& Ilic, 2015). No entanto, combater o Burnout não é apenas uma questão de reduzir o número de aspectos negativos, pois, nalgumas situações não há muito que se possa fazer nestes aspectos. Assim, na maioria das vezes é mais eficaz pensar em estratégias para aumentar o número de aspectos positivos, nomeadamente os que possam estar relacionados com o trabalhador (Maslach \& Leiter, 2008).
Deste modo, para melhorar o envolvimento no trabalho e para proteger os enfermeiros e outros profissionais de saúde a partir do risco de desenvolver stresse e Burnout no trabalho, sugerem-se intervenções como programas de redução de stresse no trabalho e estratégias de enfrentamento para fortalecer os recursos internos dos trabalhadores a fim de promover um ambiente de trabalho saudável e satisfação no trabalho (Fiabane, Giorgi, Sguazzin \& Argentero, 2013). Além disso, pesquisas em gestão de pessoas, em especial em trabalhadores da saúde, têm destacado a importância de promover as características sadias e protetoras dos trabalhadores, por meio do mapeamento e promoção da Resiliência, para poder superar as condições adversas a que estão submetidos, como o stresse laboral elevado, e assim melhorar as condições resilientes dos trabalhadores (Belancieri \& Kahhale, 2011; Chan, Chan \& Kee, 2013; Rodrigues, Barbosa \& Chiavone, 2013).

Resiliência é compreendida como "a capacidade humana para enfrentar, vencer e sair fortalecido ou transformado por experiências de adversidade", denotando a ideia de evolução (Melillo \& Ojeda, 2005, p.15). Pessoas resilientes são capazes de, após a adversidade, recuperar o equilíbrio, tanto em termos mentais quanto emocionais, e aprender com a experiência tornando-se mais fortes e preparadas (Wagnild \& Young, 1993). Resiliência é um privilégio de alguns indivíduos, pois não se nasce resiliente nem se adquire naturalmente no desenvolvimento humano. É uma competência passível de ser aprendida e de desenvolver ao longo da existência por meio do processo interativo do sujeito com outros seres humanos e de processos intrapsíquicos. Para tal, a pessoa deve apropriar-se de sua realidade e transformála, transformando, consequentemente, a si mesmo num movimento dialético (Belancieri \& Kahhale, 2011; Melillo \& Ojeda, 2005).

A Resiliência resulta das crenças do indivíduo, podendo conduzi-lo à adaptação saudável diante das adversidades e a uma experiência que implica amadurecimento e desenvolvimento, ou seja, todo indivíduo tem uma predisposição à resiliência, que pode ser desenvolvida a partir das vivências durante toda a vida (Rodrigues et al., 2013).

Envolve estabilidade interna, consciência e flexibilidade que contribuem para o enfrentamento das adversidades presentes no contexto laboral, como as situações de alto stresse, e auxilia na redução do Burnout. 
Esta questão torna- se importante pois o contexto de trabalho de enfermagem é uma situação desafiadora que muito provavelmente não se modificará brevemente.

Assim, Resiliência contribui para que os enfermeiros consigam responder ao alto nível de stresse de forma a protegerem-se contra consequências prejudiciais (Rushton, Batcheller, Schroeder \& Donohue, 2015). Além disso, a Resiliência tem sido reconhecida como aspecto importante na promoção e manutenção da saúde mental e emocional, podendo reduzir a intensidade do stresse e diminuir sinais emocionais negativos, como ansiedade, depressão ou raiva, para além de ajudar a enfrentar as adversidades (Melillo \& Ojeda, 2005). A Resiliência parece então estar associada ao stresse, mas no contexto laboral pode aumentar a adaptação individual do enfermeiro nas situações stressantes e passiveis de levar ao Burnout (Barlach, Limongi-França \& Malvezzi, 2008; Fontes \& Azzi, 2012; Lee et al., 2015). O objetivo deste estudo foi conhecer os níveis de Resiliência e Burnout de enfermeiros que atuavam em hospitais públicos da área metropolitana do Porto e sua variação em função de caracteristicas sociodemográficas e profissionais, bem como a relação entre estas duas variáveis no sentido de verificar se a Resiliência se constitui como protetora do Burnout, e desta forma, pode ser utilizada na promoção da saúde mental e ocupacional dos enfermeiros.

\section{METODOLOGIA}

\section{Participantes}

O presente estudo insere-se numa metodologia quantitativa, do tipo transversal, exploratório e descritivo. A amostra foi constituída por 200 enfermeiros que trabalhavam em hospitais públicos da área metropolitana do Porto, maioritariamente (70\%) do sexo feminino, com idades entre 25 e 57 anos (média de 33,8 anos e $\mathrm{DP}=7,6)$, sendo $43 \%$ casados ou em união de facto e $36 \%$ com filhos.

Em relação às características profissionais, $82 \%$ tem apenas a licenciatura (e restantes pós-graduação), 76\% tem um vínculo definitivo (e restantes vinculo precário), $80 \%$ trabalha em turno rotativo (e restantes em turno fixo), tendo em média 10,6 anos de experiência na profissão $(\mathrm{DP}=7,7)$ e em média de 6,6 anos de tempo na instituição $(\mathrm{DP}=6,3)$.

\section{Instrumentos}

Foram utilizados três questionários que permitiram efetuar a caracterização sociodemográfica e profissional, bem como identificar os níveis de resiliência e de Burnout.

O questionário de Caracterização Sociodemográfica e Profissional incluiu questões sobre idade, sexo, estado civil, existência de filhos, habilitações, vínculo de trabalho, turno, tempo de experiência na profissão e tempo de trabalho na instituição.

A Escala de Resiliência (adaptado de Pesce et al., 2005, do português do Brasil para português de Portugal; sendo a versão original de Wagnild \& Young, 1993) avalia o grau individual de Resiliência, considerada uma característica de personalidade positiva que aumenta a adaptação individual. Possui 25 itens descritos de forma positiva com resposta tipo Likert variando de 1 (discordo totalmente) a 7 (concordo totalmente) que avaliam 2 fatores (Competências Pessoais e Aceitação de si mesmo e da vida). Os scores da escala são calculados pela média e oscilam de 1 a 7 valores, com valores médios altos indicando elevada resiliência. Não existindo consenso em relação ao número de fatores entre os vários autores sobre a Escala de Resiliência, optamos por conhecer o fenómeno utilizando os dois fatores da escala original.

É ainda possível calcular um score total, com valores que podem variar entre 25 e 175, indicando os resultados mais elevados uma maior resiliência. Além disso, os autores originais consideram que um valor abaixo dos 121 é considerado indicativo de reduzida resiliência, entre 121 e 145 é considerado como "resiliência moderada, e acima dos 145 é considerado moderadaelevada a resiliência elevada.

Para avaliar o Burnout foi utilizado o Maslach Burnout Inventory, Human Services Survey (versão portuguesa de Marques-Pinto \& Picado, 2011; sendo a versão original de Maslach \& Jackson, 1986), com 22 itens que avaliam as três dimensões do Burnout (exaustão emocional, despersonalização e realização pessoal), numa escala de Likert de sete pontos que varia entre 0 ("Nunca") e 6 ("Todos os dias"). De acordo com os autores, a existência simultanea de pontuações elevadas nas dimensões exaustão emocional e despersonalização e pontuações baixas na realização pessoal indicam a presença de Burnout. 
É de realçar que a versão original do Maslach Burnout Inventory, bem como a sua adaptação para alguns países (ex: Espanha) apresenta pontes de corte definidores de classificações de baixo, moderado ou elevado nível. Contudo, foram calculadas para os scores totais de cada dimensão ou do Burnout total, estão actualmente desatualizadas e não existem para Portugal. Assim, utilizamos o critério recente de Maroco e colaboradores (2016, p.27), que, numa amostra nacional de enfermeiros e médicos define as seguintes categorias: "sem burnout/burnout reduzido para scores médios inferio $\neg$ res a dois; burnout moderado para scores médios entre 2 e 3; e burnout elevado para scores médios superiores ou iguais a três".

\section{Procedimentos}

Foram cumpridos requesitos éticos inerentes ao processo investigativo nomeadamente, o consentimento informado aos participantes e à Comissão de Ética da Escola Superior de Enfermagem do Porto para a realização do estudo (integrado no Projeto INT-SO).

Para a aplicação dos questionários foi utilizado a técnica bola de neve (snowball), com contato inicial com os enfermeiros do curso de especialização da Escola Superior de Enfermagem do Porto (ESEP), que em seguida contactaram outros colegas nos seus locais de trabalho, disponibilizando os questionários impressos, bem como a declaração de consentimento informado a todos os que voluntariamente aceitaram participar no estudo. Todos os participantes foram esclarecidos, por escrito, sobre os objetivos da investigação e dada garantia da confidencialidade e do anonimato, sendo os questionários devolvidos, em envelopes, aos enfermeiros que tiveram o contacto inicial com o estudo, os quais, posteriormente, os fizeram chegar à ESEP. A recolha de dados decorreu entre maio e dezembro de 2014, sem contacto direto entre participantes e investigadores.

Os dados foram analisados com recurso do programa IBM-SPSS versão 21. A estatística descritiva foi utilizada para descrever as características das variáveis sociodemográficas e profissionais da amostra, enquanto a correlação R de Pearson foi usada para as correlações entre Resiliência e Burnout. Para a comparação de médias foi utilizado o teste não paramétrico Mann Whitney-U, e no caso de encontrarmos diferenças significativas, foi ainda efetuado o teste $\mathrm{t}$-de Student para amostras independentes, por facilidade de interpretação das médias por grupos comparados.
Para a análise de regressão foram cumpridos os pressupostos para a sua utilização, optando-se pelo método Enter, colocando por blocos, respetivamente, as variáveis sociodemográficas, em seguida as variáveis profissionais e em seguida as dimensões da resiliência, tendo as dimensões do Burnout como variáveis a ser predictas pelos blocos já enunciados.

\section{RESULTADOS}

A análise dos dados (Tabela 1) evidencia que os participantes do estudo apresentaram moderada exaustão emocional, baixos valores de despersonalização e elevados valores de realização pessoal, de acordo com os limites sugeridos por Maroco e colaboradores (2016), inferindo a ausência de Burnout. Assim, tendo em consideração as categorias propostas por estes autores, no que se refere ao total de Burnout, varia entre 0,06 e 4,20, com uma média de $1,64(\mathrm{DP}=0,75)$, o que configura Burnout reduzido. Em termos de percentagens, encontrou-se 70\% da amostra sem Burnout ou com Burnout reduzido, 25\% com Burnout moderado e apenas 5\% com Burnout elevado. Em relação a Resiliência, ambos fatores (competências pessoais e aceitação de si mesmo e da vida) apresentaram escores elevados, indicativos de elevado grau de resiliência, sendo o fator competências pessoais o que apresenta o valor mais elevado. De acordo com os valores limite do total de Resiliência, esta variou entre 59 e 168, com uma média de 135,5 $(\mathrm{DP}=15,89)$, o que configura Resiliência moderada mas próxima já da passagem para Resiliência moderada/ elevada. Em termos de percentagens, $12 \%$ da amostra apresenta Resiliência reduzida, 63\% apresenta Resiliência moderada, e $25 \%$ Resiliência moderada/elevada a elevada. Nesse sentido, os resultados mostram que os enfermeiros que participaram desse estudo apresentam ausência de Burnout e elevado grau de resiliência.

Além disso, é possível observar (Tabela 1) algumas correlações estatisticamente significativas entre as dimensões do Burnout e os fatores da Resiliência, como a correlação negativa entre a exaustão emocional e as competências pessoais e a aceitação de si mesmo e da vida, ou seja, quanto menor as competências pessoais e a aceitação de si mesmo e da vida maior a exaustão emocional no trabalho. Observa-se também a correlação positiva entre a realização pessoal e as competências pessoais e a aceitação de si mesmo e da vida, ou seja, quanto maior as competências pessoais e a aceitação de si mesmo e da vida maior a realização pessoal no trabalho. 
Não há correlação estatísticamente significativa entre as competências pessoais e a aceitação de si mesmo e a despersonalização. No que se refere à idade, tempo de experiência na profissão e tempo de experiência na instituição, não se encontraram correlações significativas destas com as dimensões do Burnout e da Resiliência.

Tabela 1 - Médias, Desvios-Padrão e Correlações de Pearson entre Burnout, Resiliência e Variáveis Sociodemográficas e Profissionais

\begin{tabular}{|c|c|c|c|c|c|c|c|}
\hline $\begin{array}{l}\text { Variáveis } \\
\text { (escala) }\end{array}$ & M & $\mathrm{DP}$ & 1.EE & 2.DP & 3.RP & 4.CP & 5.AV \\
\hline \multicolumn{8}{|c|}{ Dimensões do Maslach Burnout Inventory HSS (0-6) } \\
\hline $\begin{array}{l}\text { 1. Exaustão } \\
\text { Emocional }\end{array}$ & 2,90 & 1,22 & & & & & \\
\hline $\begin{array}{l}\text { 2.Desper- } \\
\text { sonalização }\end{array}$ & 1,17 & 1,12 & $426^{\star *}$ & & & & \\
\hline $\begin{array}{l}\text { 3.Realização } \\
\text { Pessoal }\end{array}$ & 4,46 & 0,85 &,$- 241^{\star *}$ &,$- 189^{\star *}$ & & & \\
\hline \multicolumn{8}{|c|}{ Dimensões da Escala de Resiliência (1-7) } \\
\hline $\begin{array}{l}\text { 4.Competên- } \\
\text { cias Pessoais }\end{array}$ & 5,74 & 0,61 &,$- 195^{* *}$ &,- 134 & $446^{\star *}$ & & \\
\hline $\begin{array}{l}\text { 5.Aceitação } \\
\text { de si mesmo } \\
\text { e da vida }\end{array}$ & 4,95 & 0,76 &,$- 292^{\star *}$ &,- 056 &, $330^{* *}$ &, $579^{* *}$ & \\
\hline Idade & & &,- 031 &,- 075 & ,002 &,- 032 & 035 \\
\hline $\begin{array}{l}\text { Tempo de } \\
\text { experiência } \\
\text { na profissão }\end{array}$ & & &,- 107 &,- 133 & 033 &,- 011 &, 044 \\
\hline $\begin{array}{l}\text { Tempo de } \\
\text { experiência } \\
\text { na institu- } \\
\text { ição }\end{array}$ & & & 018 &,- 039 &,- 015 &,- 023 & ,035 \\
\hline
\end{tabular}

${ }^{\star} \mathrm{p} \leq 0.05{ }^{\star *} \mathrm{p} \leq 0.01$

Em relação à análise comparativa, foram efetuadas comparações para Burnout e Resiliência em função das variáveis sociodemográficas e profissionais, não se tendo encontrado diferenças estatisticamente significativas no que se refere ao sexo, existência de filhos, habilitações e vínculo de trabalho. Contudo, a variável turno apresentou resultado estatisticamente significativo ( Tabela 2) ao nível da despersonalização, apresentando valores mais elevados nos trabalhadores de turno rotativo em relação ao de turno fixo, conforme análise complementar do teste-t Student após teste não paramétrico de Mann-Whitney. No que se refere ao estado civil, apenas se encontraram diferenças significativas na dimensão da Resiliência competências pessoais, com os casados a apresentarem valores inferiores (embora sem diferenças através do test $\mathrm{t}$-Student).
Tabela 2 - Análise Comparativa em Função do

Turno e do estado civil

\begin{tabular}{|c|c|c|c|c|c|}
\hline $\begin{array}{l}\text { Dimensões } \\
\text { (escala) do } \\
\text { Burnout e } \\
\text { Resiliência }\end{array}$ & $\begin{array}{l}\text { Turno } \\
\text { fixo } \\
(\mathrm{N}=40)\end{array}$ & $\begin{array}{l}\text { Turno } \\
\text { rotativo } \\
(\mathrm{N}=160)\end{array}$ & t-Student & $\begin{array}{l}\mathrm{p} \\
\mathrm{t}-\mathrm{Stu}- \\
\text { dent }\end{array}$ & $\begin{array}{l}\text { p Mann- } \\
\text { Whit- } \\
\text { ney-U }\end{array}$ \\
\hline $\begin{array}{l}\text { Exaustão } \\
\text { Emocional } \\
(0-6)\end{array}$ & 2,72 & 2,94 & $-1,039$ & 300 & ,374 \\
\hline $\begin{array}{l}\text { Despersonal- } \\
\text { ização }\end{array}$ & ,79 & 1,27 & $-2,438$ &, $016^{\star}$ &, $009^{* *}$ \\
\hline $\begin{array}{l}\text { Realização } \\
\text { Pessoal }\end{array}$ & 4,67 & 4,41 & 1,692 & ,092 & ,092 \\
\hline $\begin{array}{l}\text { Competências } \\
\text { Pessoais (1-7) }\end{array}$ & 5,83 & 5,71 & 1,071 & ,285 & ,349 \\
\hline \multirow{2}{*}{$\begin{array}{l}\text { Aceitação de } \\
\text { si mesmo e da } \\
\text { vida }\end{array}$} & 5,03 & 4,93 & ,694 & ,489 & ,719 \\
\hline & $\begin{array}{l}\text { Não } \\
\text { casado } \\
(\mathrm{N}=114)\end{array}$ & $\begin{array}{l}\text { Casado ou } \\
\text { união facto } \\
(\mathrm{N}=86)\end{array}$ & t-Student & $\begin{array}{l}\mathrm{p} \\
\mathrm{t}-\mathrm{Stu}- \\
\text { dent }\end{array}$ & $\begin{array}{l}\text { p Mann- } \\
\text { Whit- } \\
\text { ney-U }\end{array}$ \\
\hline $\begin{array}{l}\text { Exaustão } \\
\text { Emocional }\end{array}$ & 2,88 & 2,93 &,- 318 & ,751 & ,801 \\
\hline $\begin{array}{l}\text { Despersonal- } \\
\text { ização }\end{array}$ & 1,16 & 1,18 &,- 154 & ,878 & ,666 \\
\hline $\begin{array}{l}\text { Realização } \\
\text { Pessoal }\end{array}$ & 4,42 & 4,52 &,- 807 & ,421 & ,368 \\
\hline $\begin{array}{l}\text { Competências } \\
\text { Pessoais }\end{array}$ & 5,80 & 5,65 & 1,754 & ,081 &, $016^{*}$ \\
\hline $\begin{array}{l}\text { Aceitação de } \\
\text { si mesmo e da } \\
\text { vida }\end{array}$ & 5,00 & 4,88 & 1,081 & ,281 & , 105 \\
\hline
\end{tabular}

A análise de regressão Enter (Tabela 3) revelou que as variáveis individuais (habilitações, sexo, estado civil, idade e existência de filhos) e profissionais (vinculo, turno, anos de experiência e anos na instituição) não são preditoras do Burnout, enquanto a Resiliência explica negativamente $8 \%$ da exaustão emocional e positivamente $26 \%$ da realização pessoal. A despersonalização não tem preditores significativos.

Tabela 3 - Regressão (Enter) das Variáveis Individuais,

Profissionais e Resilência no Burnout

\begin{tabular}{|c|c|c|c|c|}
\hline $\begin{array}{c}\text { Variável } \\
\text { Dependente }\end{array}$ & Variáveis Preditoras & $\mathrm{R} 2$ & R2 change & $\mathrm{F}($ sig $)$ \\
\hline \multirow{3}{*}{$\begin{array}{l}\text { Exaustão } \\
\text { Emocional }\end{array}$} & Individuais & 015 & 015 & ,514 (,766) \\
\hline & Profissionais & ,054 & ,039 & $1,041(, 410)$ \\
\hline & Resiliência & , 135 & ,082 & $2,319\left(, 011^{*}\right)$ \\
\hline \multirow{3}{*}{$\begin{array}{c}\text { Realização } \\
\text { Pessoal }\end{array}$} & Individuais &, 032 & ,032 & $1,115(, 354)$ \\
\hline & Profissionais & .074 & ,042 & $1,471(, 163)$ \\
\hline & Resiliência & 084 & ,010 & $1,363(, 195)$ \\
\hline \multirow{3}{*}{$\begin{array}{c}\text { Realização } \\
\text { Pessoal }\end{array}$} & Individuais & ,044 & 044 & $1,549(, 177)$ \\
\hline & Profissionais & 069 & ,025 & $1,355(, 213)$ \\
\hline & Resiliência & ,287 & ,262 & $\begin{array}{c}7,336 \\
\left(, 000^{* * *}\right)\end{array}$ \\
\hline
\end{tabular}




\section{DISCUSSÃO}

O comportamento resiliente advém em regular o controle dos impulsos frente às condições stressantes presentes no quotidiano. Indivíduos com maior grau de resiliência tendem a controlar os impulsos e a ter alta regulação das emoções, indivíduos contrários tendem a ter baixa regulação das emoções, a serem impulsivos e agirem de acordo com essa impulsividade, o que gera dificuldade no controle dos impulsos e uma redução da capacidade resiliente (Belancieri \& Kahhale, 2011). O fortalecimento do comportamento resiliente é resultante da capacidade de que se pode conviver com as adversidades e contrariedades presentes no ambiente de trabalho. Para tal, o indivíduo recorre à flexibilidade, uma das características da resiliência, no enfrentamento das situações de tensão, pressão e stresse (Barlach et al., 2008; Fontes \& Azzi, 2012). Os enfermeiros deste estudo obtiveram elevada pontuação nos fatores que compõem a resiliência e na realização pessoal e menores pontuações na exaustão emocional e na despersonalização. Num estudo recente em enfermeiras de unidade de terapia intensiva, também foi observado ausência de Burnout e elevado grau de Resiliência. Além disso, as enfermeiras que pontuaram mais na dimensão realização pessoal apresentaram maior pontuação na escala de resiliência (Rushton et al., 2015). Nesse mesmo estudo, foi possível encontrar uma correlação negativa entre a resiliência e as dimensões exaustão emocional e despersonalização, mas positiva com realização pessoal. Através de regressão linear evidenciou-se que maior resiliência protegia enfermeiros da exaustão emocional e contribuía para a realização pessoal. Os autores concluiram que a resiliência é uma variável envolvida na redução da vulnerabilidade dos enfermeiros à exaustão emocional, e que elevada pontuação da resiliência associa-se a redução do stresse, sendo um fator de proteção contra o Burnout (Rushton et al., 2015). Também Maroco e colaboradores (2016), num estudo nacional encontraram moderado Burnout em enfermeiros, embora sem muitas diferenças significativas entre regiões, tipo de instituição de saúde ou variaveis individuais.

Pode ainda dizer-se que trabalhadores que apresentam comportamentos resilientes ao buscarem o equilíbrio emocional e a conhecerem-se a si próprios, preparamse técnico-cientificamente de acordo com as demandas laborais, antecedem as dificuldades, constroem novos sentidos e ações e elaboram estratégias de enfrentamento ante as dificuldades cotidianas a que estão submetidos como déficit de recursos humanos, de recursos materiais, finitude e sofrimento dos pacientes, conflito interpessoal, entre outros (Belancieri \& Kahhale, 2011).
Num outro estudo realizado entre os profissionais de saúde de unidade de terapia intensiva pediátrica, ao avaliar resiliência observaram que os indivíduos com scores mais altos de Resiliência têm a percepção de maior trabalho em equipe e que aqueles com mais de 7 anos de experiência pontuaram mais em Resiliência (Lee et al., 2015). O pressuposto é que ao elevar o grau de Resiliência dos enfermeiros isso afetará, consequentemente, a cultura organizacional, a criação de ambientes de trabalho mais saudáveis, e impulsionará os enfermeiros a envolver-se nos arranjos organizacionais (Rushton et al., 2015). Ou seja, ao investigar a Resiliência entre enfermeiros, visa-se focar nos aspectos saudáveis, desenvolvendo flexibilidade para enfrentar as adversidades e, consequentemente, promover a saúde mental e ocupacional entre os profissionais de enfermagem (Rushton et al., 2015).

A análise comparativa evidenciou que a variável turno apresentou resultado estatisticamente significativo ao nível da despersonalização, apresentando valores mais elevados nos profissionais de turno rotativo em relação ao de turno fixo. Portanto, trabalhar em turnos rotativos dificulta a adaptação e o vínculo do trabalhador, comprometendo o comportamento resiliente e tornando-o mais vulnerável ao Burnout. Recursos adicionais para promoção da resiliência podem ser otimizados, como realizar uma pausa de pacientes stressantes, retirar o profissional do serviço depois da morte do seu paciente, apoio e cuidados para o pessoal, atividades sociais fora do hospital, entre outros. Portanto, para a promoção da resiliência é necessário haver um conjunto de ações para satisfazer as necessidades pessoais e profissionais de todos os membros da equipe em cada unidade (Lee et al., 2015). Por outro lado, é importante estabelecer um clima de confiança em que os profissionais se sentem livres para falar, para dizer o que precisa ser dito e não apenas o que se queria ser ouvido (Coates \& Howe, 2015). Por fim, a resiliência resulta das crenças do indivíduo, podendo conduzi-lo à adaptação saudável diante das adversidades e a uma experiência que implica amadurecimento e desenvolvimento, ou seja, o indivíduo pode desenvolver a resiliência a partir das suas vivências durante toda a vida (Rodrigues et al., 2013).

\section{CONCLUSÃO}

Os resultados obtidos neste estudo permitiram, junto de enfermeiros a exercerem funções em hospitais públicos da área metropolitana do Porto, encontrar, no que se refere às médias, níveis de moderada exaustão emocional, baixos valores de despersonalização, e elevados valores de realização pessoal e de Resiliência. 
Em termos percentuais, 70\% da amostra apresenta-se sem Burnout ou com Burnout reduzido e apenas 5\% tem Burnout elevado, enquanto no que se refere à Resiliência, apenas $12 \%$ tem reduzida e $63 \%$ tem moderada. Existem correlações negativas entre exaustão emocional e resiliência, e correlações positivas entre realização pessoal e resiliência, sendo possível perceber que trabalhadores com elevado grau de Resiliência tendem a não apresentar Burnout. A análise comparativa evidenciou o turno com diferenças significativas na despersonalização, tendo valores mais elevados nos trabalhadores de turno rotativo. A resiliencia explica negativamente $8 \%$ da exaustão emocional e positivamente $26 \%$ da realização pessoal, valores significativos por oposição a características sociodemográficas e profissionais.

Apesar dos valores ainda não preocupantes em termos de Saúde Ocupacional, note-se qe existem já $5 \%$ de enfermeiros com Burnout elevado e 12\% com Resiliência reduzida, o que no atual contexto de crise sócioeconómica e de mudanças frequentes a nível laboral, constitui um alerta. Assim, intervenções sobre o ambiente de trabalho e o trabalhador deve ser pensadas, pois contribuem para a gestão do stresse no trabalho e são facilitadoras e promotoras de comportamento resiliente dos trabalhadores. A literatura demonstrou que trabalhadores resilientes tendem a ter maior desempenho e a serem mais comprometidos com o trabalho, com a organização, e consequentemente, com a prestação da assistência de enfermagem com maior qualidade. Mas para tal, é necessário ter também iniciativas institucionais resolutivas e motivadoras que promovam a saúde do trabalhador de enfermagem.

\section{IMPLICAÇÕES PARA A PRÁTICA CLÍNICA}

Este estudo demonstrou que quanto maior o nível de Resiliência menor a probabilidade de desenvolver o Burnout. Contudo, torna-se necessária uma reflexão por parte das instituições sobre alguns pontos importantes tais como: as competências pessoais e a aceitação de si mesmo e da vida que pode influenciar diretamente na exaustão emocional no trabalho; o tempo de experiência de profissão e a despersonalização; e o turno rotativo que comparativamente apresenta maior despersonalização. O conhecimento destas variáveis, com implicação no aumento ou redução da Resiliência e todas as suas consequências, permitirá às instituições construírem um plano estratégico a fim de promover a saúde mental e ocupacional do enfermeiro, o que terá reflexos diretamente na organização institucional e no cuidado ao paciente.

\section{REFERÊNCIAS BIBLIOGRÁFICAS}

Barlach, L., Limongi-França, A.C., \& Malvezzi, S. (2008). O conceito de resiliência aplicado ao trabalho nas organizações. Interamerican Journal of Psychology, 42(1), 101-112.

Belancieri, M.F., \& Kahhale, E.M. (2011). A saúde do cuidador: Possibilidades de promoção de resiliência em enfermeiros. Revista Mineira de Enfermagem, 15(1), 121-128.

Chan, A.O., Chan, Y.H., \& Kee, J.P. (2013). Exposure to crises and resiliency of health care workers in Singapore. Occupational Medicine, 63(2), 141-144.

Coates, D., \& Howe, D. (2015). Combatting staff burnout in mental health: Key managerial and leadership tasks that are fundamental to staff wellbeing and retention. Asia Pacific Journal of Health Management, 10(2), 24-32.

Davey, A., Sharma, P., Davey, S., Shukla, A., Srivastava, K., \& Vyas, S. (2016). Are the adverse psychiatric outcomes reflection of occupational stress among nurses: An exploratory study. Asian Journal of Medical Sciences, 7(1), 96-100.

Fiabane, E., Giorgi, I., Sguazzin, C., \& Argentero, P. (2013). Work engagement and occupational stress in nurses and other healthcare workers: The role of organizational and personal factors. Journal of Clinical Nursing, 22, 2614-2624.

Fontes, A.P., \& Azzi, R.G. (2012) Crenças de autoeficácia e resiliência: Apontamentos da literatura sociocognitiva. Estudos de Psicologia Campinas, 29(1), 105-114.

Khamisa, N., Oldenburg, B., Peltzer, K., \& Ilic, D. (2015). Work related stress, burnout, job satisfaction and general health of nurses. International Journal of Environmental Research on Public Health, 12, 652-666.

Lee, J., Forbes, M., Lukasiewicz, G.J., Williams, T., Sheets, A., Fischer, K., \& Niedner, M. (2015). Promoting staff resilience in the pediatric intensive care unit. American Journal of Critical Care, 24(5), 422-430. 
Maroco, J., Maroco, A. L., Leite, E., Bastos, C., Vazão, M. J., \& Campos, J. (2016). Burnout em profissionais da saúde portugueses: Uma análise a nível nacional. Acta Médica Portuguesa, 29(1), 24-30.

Marques-Pinto, A., \& Picado, L. (2011). Bem-estar e adaptação nas escolas portuguesas. Lisboa: Coisas de Ler.

Maslach, C., \& Leiter, M. P. (2008). Early predictors of job burnout and engagement. Journal of Applied Psychology, 93(3), 498-512.

Maslach, C., \& Jackson, S. E. (1986). Maslach burnout inventory (2nd ed.). Palo Alto, CA: Consulting Psychologist Press.

Melillo, A., \& Ojeda, E. (2005). Resiliência: Descobrindo as próprias fortalezas. Porto Alegre: Artmed.

Metzger, J. (2011). Mudança permanente: Fonte de penosidade no trabalho? Revista Brasileira de Saúde Ocupacional, 36(23), 12-24.
Pesce, R., Assis, S., Avanci, J., Santos, N., Malaquias, J., \& Carvalhães, R. (2005). Adaptação transcultural, confiabilidade e validade da escala de resiliência. Cadernos de Saúde Pública, 21(2), 46-448. Ramos, E., Souza, N.,

Gonçalves, F., Pires, A., \& Santos, D .M. (2014). Qualidade de vida no trabalho: Repercussões para a saúde do trabalhador de enfermagem de terapia intensiva, que tem moldado o perfil epidemiológico de adoecimento dessa categoria. Revista de Pesquisa Cuidado é Fundamental Online, 6(2), 571-583.

Rodrigues, R., Barbosa, G. \& Chiavone, P. (2013). Personalidade e resiliência como proteção contra o burnout em médicos residentes. Revista Brasileira de Educação Médica, 37(2), 245-253.

Rushton, C., Batcheller, J., Schroeder, K., \& Donohue, P. (2015). Burnout and resilience among nurses practicing in high-intensity settings. American Journal of Critical Care, 24(5), 412-421.

Wagnild, G., \& Young, H. (1993). Development and psychometric evaluation of the Resilience Scale. Journal of Nursing Measurement, 1(2), 165-178. 